\title{
Isle of Man
}

National Cancer Institute

\section{Source}

National Cancer Institute. Isle of Man. NCI Thesaurus. Code C44480.

An island in the Irish Sea, between Great Britain and Ireland. 